\title{
Prevalence of Early Laryngeal Cancer and Benign Vocal Cord Pathology among Patients Undergoing Videostroboscopy in Philippine General Hospital from 2008 to 2010
}

\author{
Ryner Jose C. Carrillo, Jan Warren A. Holgado, Melfred L. Hernandez and Rene S. Tuazon
}

Department of Otorhinolaryngology, College of Medicine and Philippine General Hospital, University of the Philippines Manila

\begin{abstract}
Objective. To determine the types and prevalences of laryngeal pathology among patients undergoing videostroboscopy in the Philippine General Hospital.

Methods. A systematic review of videostroboscopy records of patients from January 2008 to December 2010 was performed. Demographic data, risk factors, diagnosis and their association were determined.

Results. The following vocal cord pathologies were recorded for the 507 subjects who underwent videostroboscopy during the assessed time period: vocal cord nodules (17.4\%), laryngopharygeal reflux (16.6\%), vocal cord paralysis (12.8\%), cyst $(10.5 \%)$, polyp (8\%) and laryngeal cancer (6\%). Vocal cord nodules were found to be more common among professional voice users (odds ratio $=2.8$ ). Risk factors found to be associated with cancer development include age, gender (male, odds ratio 19.8) and smoking history (odds ratio $=12.7$ ).
\end{abstract}

Conclusion. The most common laryngeal pathology among patients undergoing videostroboscopy is vocal cord nodules. One out of 20 patients who underwent the procedure was given a diagnosis of laryngeal cancer.

Key Words: stroboscopy, laryngeal pathology

\section{Introduction}

Hoarseness is the most common manifestation of vocal fold movement abnormality. If hoarseness is observed to be persistent beyond two weeks, examination of the larynx is warranted to rule out vocal cord pathologies, particularly early infiltrative processes of the vocal fold. While traditional examination of the larynx using mirrors, combined with symptoms of hoarseness, are the usual means of providing a diagnosis, a permanent record of laryngeal image and adequate description of vocal fold movement is very helpful for the otolaryngologist in making appropriate management decisions. ${ }^{1}$

Corresponding author: Ryner Jose C. Carrillo, MD

Department of Otorhinolaryngology

Philippine General Hospital

University of the Philippines Manila

Taft Avenue, Ermita, Manila 1000 Philippines

Telephone: +6325264360

Email: ryner_c@yahoo.com
Videostroboscopy of the larynx allows examination of vocal fold movement during phonation. Mucosal waves of the vocal fold are demonstrated by stroboscopic lights allowing illusory frame by frame movement of the vocal fold which demonstrates viscoelastic properties of the normal vocal fold epithelium and superficial lamina propria. Decreased or absent mucosal wave changes observed may suggest severe dysplasia or carcinoma of the vocal cords. ${ }^{2}$ Laryngovideostroboscopy is the standard diagnostic test prior to determining biopsy of the vocal cords for benign and early malignant pathology. ${ }^{3}$

The Laryngology Section of the Department of Otorhinolaryngology, Philippine General Hospital has been performing videostroboscopy on pay and charity patients who present with voice and swallowing symptoms since 2000. Videostroboscopy assessments are generally based on vocal cord image and characteristics, such as glottal closure, phase closure, vertical level, amplitude, mucosal wave, vibratory behavior, phase symmetry and periodicity. Ventricular folds and arytenoids are also assessed. This paper determines the prevalence of laryngeal pathologies among PGH patients who underwent videostroboscopy over a three - year period.

\section{Methods}

Videostroboscopy recordings from Jan 2008 to Dec 2010 obtained using Kay Elemetrics digital strobe 9200B machine from the voice lab of the Department of Otorhinolaryngology, Philippine General Hospital, and corresponding assessments, were reviewed. Variables studied include age, sex, profession, smoking history, alcohol intake, chief complaint and diagnosis. Patients with presumptive diagnosis of cancer were reviewed and definitive biopsy results were obtained. Data was encoded and analyzed using Intercooled Stata 9.2 for Windows (StataCorp LP). Mean, proportions, crude odds ratios and adjusted odds ratios were calculated. Patient records were anonymized; the study was done in compliance with the Declaration of Helsinki and Good Clinical Practice.

\section{Results}

A total of 507 videostroboscopy examinations were performed from 2008 to 2010. Demographic data is 
summarized in Table 1. Significantly, $45.4 \%$ of examined patients were male; $40.5 \%$ were smokers, $45 \%$ had alcohol intake, and 19\% were professional voice users (e.g., teachers, singers). (Table 1). The most common chief complaint among patients was hoarseness $(77.6 \%)$ followed by globus sensation $(5.9 \%)$. (Table 2$)$ The common diseases observed were vocal cord nodules (17.4\%), laryngopharyngeal reflux $(16.6 \%)$, vocal cord paresis $(12.8 \%)$, cyst $(10.4 \%)$, polyp $(8.1 \%)$ and presumptive cancer $(6.1 \%)$. Definite laryngeal cancer verified by biopsy is $1.8 \%$. (Table 3 )

Table 1. Demographics of patients undergoing videostroboscopy $(\mathrm{N}=507)$

\begin{tabular}{ll}
\hline Age (mean) & 46.5 years old (range $11-84$ years old) \\
Gender (male) & $45.4 \%(230 / 507)$ \\
Smoker & $40.5 \%(189 / 462)$ \\
Alcohol intake & $45 \%(203 / 447)$ \\
Professional voice user & $19.3(74 / 383)$ \\
\hline
\end{tabular}

Table 2. Chief complaints of patients undergoing videostroboscopy

\begin{tabular}{ll}
\hline Hoarseness & $77.6 \%(392 / 505)$ \\
Globus sensation & $5.9 \%(30 / 505)$ \\
Dysphonia & $4.8 \%(24 / 505)$ \\
Dysphagia & $4.6 \%(23 / 505)$ \\
Throat clearing & $4.4 \%(22 / 483)$ \\
Laryngeal mass & $0.6 \%(3 / 502)$ \\
Neck mass & $0.6 \%(3 / 505)$ \\
\hline
\end{tabular}

Table 3. Presumptive diagnosis of patients undergoing videostroboscopy

\begin{tabular}{ll}
\hline Vocal cord nodule & $17.4 \%(88 / 507)$ \\
Laryngopharyngeal reflux & $16.6 \%(84 / 507)$ \\
Vocal cord paralysis or paresis & $12.9 \%(65 / 507)$ \\
Vocal cord cyst & $10.4 \%(53 / 507)$ \\
Vocal cord polyp & $8 \%(41 / 507)$ \\
Laryngeal cancer & $6.1 \%(31 / 507)$ \\
$\quad$ Early glottic cancer & $5.3 \%(27 / 507)$ \\
$\quad$ Glottic cancer with fixed cord & $0.8 \%(4 / 507)$ \\
$\quad$ Glottic cancer by biopsy & $1.8 \%(9 / 507)$ \\
Tuberculosis of the larynx & $4.9 \%(25 / 507)$ \\
Non specific dysphonia & $4.5 \%(24 / 505)$ \\
Sulcus vocalis & $3.2 \%(16 / 507)$ \\
Papilloma & $3.0 \%(15 / 507)$ \\
Laryngitis & $2.8 \%(14 / 507)$ \\
Erythroplakia, Leukoplakia, Keratosis & $2.8 \%(14 / 507)$ \\
Post operative stroboscopy & $2.4 \%(12 / 507)$ \\
Presbylarynx & $1.2 \%(6 / 507)$ \\
Supraglottic mass & $1 \%(5 / 507)$ \\
Reinke's edema & $0.8 \%(4 / 507)$ \\
Arytenoid granuloma & $0.8 \%(4 / 507)$ \\
Glottic web & $0.6 \%(3 / 507)$ \\
Supraglottic stenosis & $0.4 \%(2 / 507)$ \\
Subglottic stenosis & $0.4 \%(2 / 507)$ \\
Atrophy of the vocal cords & $0.4 \%(2 / 507)$ \\
Capillary ectasia & $0.4 \%(2 / 507)$ \\
Arytenoid dislocation & $0.2 \%(1 / 507)$ \\
Normal videostroboscopy & $6.7 \%(34 / 507)$ \\
\hline
\end{tabular}

A presumptive diagnosis of laryngeal cancer was made on 31 of 507 patients based on vocal cord morphology.
Because PGH-ORL is a referral center and caters to patients coming from various institutions, the definitive diagnosis of all patients recommended for direct laryngoscopy with biopsy is not necessarily complete. Among the 31 patients diagnosed with laryngeal cancer, 10 underwent direct laryngoscopy with biopsy under charity services in PGHORL. Nine subjects were confirmed to have Squamous Cell Carcinoma, while one was diagnosed with severe dysplasia. Initial findings show that videostroboscopy has a $90 \%$ positive predictive value for laryngeal cancer diagnosis.

\section{Discussion}

Persistent hoarseness for two weeks generally warrants referral to an otorhinolaryngologist for indirect laryngoscopy, as the pathology may possibly be worse than a simple laryngitis. ${ }^{4}$ The clinical practice guideline of 2009 by AAOHNS recommends that laryngoscopy of patients presenting with hoarseness be done when symptom duration is of a maximum of three months or anytime if there are symptoms leading to suspicion of serious disease. ${ }^{4}$ The patients referred to PGH-ORL for videostroboscopy usually have varied durations of symptoms as well as associated risk factors.

Videostroboscopy has the capacity to differentiate between voice pathologies. A rigid laryngoscope allowing larger and brighter image, combined with strobe light, provides a more detailed examination of glottic closure, mucosal wave, and amplitude to detect subtle vocal fold pathology. ${ }^{6}$ It also allows evaluation of effectiveness of treatment of patients with organic lesions of the larynx. ${ }^{7}$ Vocal cord nodules were generally more common (odds ratio of 2.8) among professional voice users, such as teachers, preachers, call center agents, as they are generally more subject to voice abuse (Table 4). Small vocal cord nodules will generally require voice rest and rehabilitation. Laryngopharyngeal reflux will require diet modification and proton pump inhibitors. While inter-observer variations do occur, the most important utility of the diagnostic test is to determine which patients need to undergo surgical biopsy as treatment to improve voice or as diagnostic test to rule out cancer. ${ }^{8,9}$ Vocal cord cysts, polyps and cancer will require direct laryngoscopy with microscope and corresponding biopsy under general anesthesia to provide definite diagnosis and to treat underlying pathology. ${ }^{10}$ Twenty-one (21) subjects of 507 patients were given a presumptive diagnosis of laryngeal cancer based on vocal cord morphology and patient profile, and were advised to undergo direct laryngoscopy with biopsy to establish a more definitive diagnosis. However, data from only 10 charity patients were available for analysis. Of these, nine cases were determined to be squamous cell carcinoma and one was found to have severe dysplasia after biopsy.

The most important risk factors for cancer include age, male gender and history of smoking and alcohol. All 9 
subjects with cancer by biopsy were male and non professional voice users. Multiple logistic regression analysis showed that age is the most important risk factor for cancer of the larynx. (Table 5) This study allows association of diagnosis of cancer and smoking. Previous studies have shown that the induction time and dose response relationship between smoking and cancer of the larynx establishes causality between smoking and cancer of the larynx. ${ }^{11}$ In this study, association between smoking and alcohol versus the diagnosis of cancer is observed as well. It should be noted, however, that possible examiner bias may affect videostroboscopy readings. For example, if a patient has a history that includes smoking and alcohol, and other risk factors such as age and sex, the examiner may be more likely to come up with the presumptive diagnosis of cancer and be recommended for direct laryngoscopy and biopsy for confirmation. Multivariate analysis showed that age is the most important of the risk factors.

Table 4. Logistic regression analysis for vocal cord nodules and risk factors

\begin{tabular}{lll}
\hline \multicolumn{1}{c}{ Risk factor } & $\begin{array}{c}\text { Odds Ratio, crude } \\
(\mathbf{9 5 \%} \mathbf{~ C I})\end{array}$ & $\begin{array}{c}\text { Odds Ratio, adjusted } \\
\mathbf{( 9 5 \% ~ C I )}\end{array}$ \\
\hline Age & $0.98(0.96-0.99)$ & $0.98(0.96-1.00)$ \\
Male gender & $0.3(0.15-0.47)$ & $0.45(0.20-1.00)$ \\
Smoking history & $0.4(0.24-0.72)$ & $0.98(0.43-2.24)$ \\
Alcohol intake & $0.4(0.24-0.70)$ & $0.59(0.27-1.29)$ \\
Occupation & $3.3(1.8-5.9)^{*}$ & $2.8(1.5-5.4)^{*}$ \\
\hline * Positive association between vocal cord nodules and professional voice use
\end{tabular}

Table 5. Logistic regression analysis for laryngeal cancer verified by biopsy (9 Squamous cell carcinoma) and risk factors

\begin{tabular}{lll}
\hline \multicolumn{1}{c}{ Risk factor } & Odds Ratio, crude (95\% CI) & \multicolumn{1}{c}{$\begin{array}{c}\text { Odds Ratio, } \\
\text { adjusted (95\% CI) }\end{array}$} \\
\hline Age & $1.04(0.99-1.09)^{*}$ & $1.02(0.96-1.08)^{*}$ \\
Male gender & All cancer patients are male* & $\mathrm{NA}^{* *}$ \\
Smoking history & $14.8(2.5-87.3)^{*}$ & $\mathrm{NA}^{* *}$ \\
Alcohol intake & $13.7(2.19-85.4)^{*}$ & $\mathrm{NA}^{* *}$ \\
Occupation & All cancer patients are non- & $\mathrm{NA}^{* *}$ \\
& professional voice users* &
\end{tabular}

${ }^{*}$ Clinically and statistically important risk factors

**In the study group, sex, smoking, alcohol intake and occupation were dropped in the multiple logistic regression analysis

It is interesting to note that among treatment - seeking patients in other countries, the prevalence of laryngeal pathology diagnosed using videostroboscopy is higher in females than males. The most common pathology is usually functional dysphonia, followed by vocal fold nodules (prevalence rates of $41 \%$ and $15 \%$ of the examined patients, respectively. ${ }^{5}$ In contrast, the most common pathology diagnosed in the PGH ORL videostroboscopy unit is vocal cord nodule (found in $17.4 \%$ of examined patients). Only 1 out of 5 patients seen were voice professionals in the local setting, compared to $41 \%$ of the voice treatment seeking population abroad. ${ }^{5}$ The discrepancies between local and overseas data may be due to variable protocols and risk factors, as well as differing definitions of disease. The videostroboscopy setup in PGH-ORL is unique in that all patients seen in out-patient services undergo laryngoscopy indirectly using mostly mirror examination with option of rigid or $f$ lexible laryngeal endoscopes when the instrument is available. Patients presenting with laryngeal symptoms, e.g., hoarseness and globus pharyngeus, are referred to the videostroboscopy unit for detailed and documented examination of the vocal fold mucosa. The findings contribute to the otolaryngologist's decision making on who will undergo a more invasive procedure for both benign and malignant pathologies. In the patients with presumptive diagnosis of cancer, videostroboscopy also provides detailed visualization of mucosal wave and gross vocal fold movement. It differentiates between glottic cancer stages. A prioritized elective direct laryngoscopy and biopsy under microscopy and formal endoscopy is done in patients with laryngeal cancer. This protocol allows early detection and management of laryngeal cancer in situation of limited hospital ward capacity. Early laryngeal cancer may undergo radiotherapy or conservation laryngectomy to attain cure with voice preservation. Cancer of the larynx with airway compromise usually warrants admission for emergent biopsy and securing of airway and generally does not undergo videostroboscopy. The ideal situation and future direction of the department is to have a database and unified system wherein all patient files are integrated with recorded endoscopy findings on first consult. This will allow a more direct assessment of protocol effectiveness and efficiency.

\section{References}

1. Bless DM, Swift E. Stroboscopy, New diagnostic techniques and applied physiology. In: Fried MP, ed. The larynx, a multidisciplinary approach, $2^{\text {nd }} \mathrm{ed}$. Missouri: Mosby-Yearbook, Inc.; 1996. pp. 81-100.

2. Gamboa J, Echeverria L, Molina B, Cobeta I. Stroboscopic assessment of chronic laryngitis. Acta Otorrinolaringol Esp. 2006; 57(6):266-9.

3. Bigenzahn W, Steiner E, Denk DM, Turetschek K, Frühwald F. Stroboscopy and imaging in interdisciplinary diagnosis of early stages of laryngeal carcinoma. Radiologe. 1998; 38(2):101-5.

4. Schwartz SR, Cohen SM, Dailey $\mathrm{SH}$, et al. Clinical practice guidelines: hoarseness (dysphonia). Otolaryngol Head Neck Surg. 2009; 141(3 Suppl 2): S1-S31.

5. Van Houtte E, Van Lierde K, D'Haeseleer E, Claeys S. The prevalence of laryngeal pathology in a treatment-seeking population with dysphonia. Laryngoscope. 2010; 120(2):306-12.

6. Casiano RR, Zaveri V, Lundy DS. Efficacy of videostroboscopy in the diagnosis of voice disorders. Otolaryngol Head Neck Surg. 1992; 107(1):95100.

7. Kluch W, Olszewski J. The use of laryngostroboscopy in diagnostics and results of evaluation of treatment of patients with organic lesions in the larynx. Otalaryngol Pol. 2009; 63(7):11-5.

8. Rosen CA. Stroboscopy as a research instrument: development of a perceptual evaluation tool. Laryngoscope. 2005; 115(3):423-8.

9. Cutler JL, Cleveland T. The clinical usefulness of laryngeal videostroboscopy and the role of high speed cinematography in laryngeal evaluation. Curr Opin Otolaryngol Head Neck Surg. 2002. 10(6); 462-6.

10. Colton RH, Caspar FK. Understanding Voice Problems, $3^{\text {rd }}$ ed. Philadelphia: Lippincott Williams and Wilkins; 2006. pp. 12-63, 100-115.

11. Terrell JE, Fisher SG, Wolf GT for Veterans Affair Laryngeal Cancer Study Group. Long-term quality of life after treatment of laryngeal cancer. Arch Otolaryngol Head Neck Surg. 1998; 124(9):964-71. 